\title{
Diabetic cardiomyopathy: where we are and where we are going
}

\author{
Wang-Soo Lee ${ }^{1}$ and Jaetaek Kim²
}

Divisions of ${ }^{1}$ Cardiology and ${ }^{2}$ Endocrinology and Metabolism, Department of Internal Medicine, Chung-Ang University College of Medicine, Seoul, Korea

Received: June 27, 2016 Accepted: January 8, 2017

\author{
Correspondence to \\ Jaetaek Kim, M.D. \\ Division of Endocrinology and \\ Metabolism, Department of \\ Internal Medicine, \\ Chung-Ang University Hospital, \\ 102 Heukseok-ro, Dongjak-gu, \\ Seoul o6973, Korea \\ Tel: $+82-2-6299-1397$ \\ Fax: +82-2-6299-1390 \\ E-mail:jtkim@cau.ac.kr
}

The global burden of diabetes mellitus and its related complications are currently increasing. Diabetes mellitus affects the heart through various mechanisms including microvascular impairment, metabolic disturbance, subcellular component abnormalities, cardiac autonomic dysfunction, and a maladaptive immune response. Eventually, diabetes mellitus can cause functional and structural changes in the myocardium without coronary artery disease, a disorder known as diabetic cardiomyopathy (DCM). There are many diagnostic tools and management options for DCM, although it is difficult to detect its development and effectively prevent its progression. In this review, we summarize the current research regarding the pathophysiology and pathogenesis of DCM. Moreover, we discuss emerging diagnostic evaluation methods and treatment strategies for DCM, which may help our understanding of its underlying mechanisms and facilitate the identification of possible new therapeutic targets.

Keywords: Diabetic cardiomyopathies; Heart failure; Diabetes mellitus

\section{INTRODUCTION}

The cardiovascular disease-related mortality with diabetes mellitus is $\sim 65 \%$. Therefore, diabetes mellitus is regarded as a risk equivalent to coronary heart disease [1]. Diabetic heart disease is a growing and important public health risk [2]. It affects the heart in three ways: cardiac autonomic neuropathy (CAN), coronary artery disease $(\mathrm{CAD})$ due to accelerated atherosclerosis, and diabetic cardiomyopathy (DCM) [1]. DCM is characterized by lipid accumulation in cardiomyocytes, fetal gene reactivation, and left ventricular (LV) hypertrophy, which together result in contractile dysfunction [2].

In 1881, Leyden first reported that DCM is a typical complication of diabetes mellitus. In 1888, Mayer asserted that diabetes mellitus is a metabolic disorder that can induce heart disease. Finally, the term "diabetic cardiomyopathy" was proposed by Rubler in 1972 after post- mortem studies in diabetic patients with heart failure in whom coronary disease and other structural heart diseases, hypertension, and alcohol had been ruled out as possible causes [3]. A milestone study in 2002 by Finck and colleagues [4] cast light on the transcriptional mechanisms of DCM. These researchers suggested that the transcription factor, peroxisome proliferator-activated receptor (PPAR)- $\alpha$, along with its transcriptional targets, is upregulated in the hearts of mouse models of diabetes mellitus $[2,4]$. Currently, DCM is defined as myocardial dysfunction $(\mathrm{MD})$ in patients with diabetes mellitus in the absence of hypertension and structural heart diseases such as valvular heart disease or CAD [5].

Diabetes mellitus is a well-known risk factor for the development of heart failure. The Framingham Heart Study demonstrated that the frequency of heart failure is five times greater in diabetic women and two times greater in diabetic men compared with age-matched 
control subjects [6]. Heart failure leads to a poor quality of life in affected individuals and complicates the treatment of diabetes mellitus by altering the pharmacokinetics of anti-diabetic medications. Thus, both the prompt diagnosis and early management of these patients are of utmost importance. However, DCM is poorly understood by most physicians, even cardiologists and diabetologists. Therefore, in this review we focus on the pathophysiological mechanism behind DCM and management strategies, including emerging therapeutics and diagnostic evaluation.

\section{EPIDEMIOLOGY OF DIABETIC CARDIOMYOP- ATHY}

The prevalence of heart failure among diabetic patients was as high as $19 \%$ to $26 \%$ in various clinical trials $[7,8]$. These two disease entities tend to coexist, and the impact of each condition on the other has bidirectional influences in terms of causation and outcome [9-11]. The Framingham Heart Study reported that $19 \%$ of patients with heart failure have type 2 diabetes mellitus (T2DM) and that the risk of heart failure increases 2- to 8-fold in the presence of $\mathrm{T} 2 \mathrm{DM}[10,12,13]$. Furthermore, an increase of $1 \%$ in hemoglobin $\mathrm{A} 1 \mathrm{c}(\mathrm{Hb} \mathrm{A} 1 \mathrm{c})$ levels is related to an $8 \%$ increase in the risk of heart failure, independently of age, body mass index, blood pressure, and the presence of CAD. This suggests that the risk of heart failure is controlled by factors unique to T2DM, such as hyperglycemia and insulin resistance $[10,12,13]$. Conversely, a $1 \%$ reduction in HbAic levels is related to a $16 \%$ reduced risk of developing heart failure and poor outcomes [10]. This bidirectional interaction has provided evidence to support the existence of DCM as a distinct clinical condition, and suggests that the presence of diabetes mellitus might independently increase the risk of heart failure [9].

The prevalence of DCM is not yet clear because of a lack of large study outcomes from different populations with diabetes mellitus. The prevalence of diastolic dysfunction in patients with $\mathrm{T} 2 \mathrm{DM}$ was up to $30 \%$ in some studies [14]. However, there are other studies that reported a prevalence rate as high as $40 \%$ to $60 \%$ [15]. A recent major prospective study examining the prevalence of heart failure and MD in patients with chronic
( $\geq 10$ years) type 1 diabetes mellitus (TiDM) showed a prevalence of $3.7 \%$ and $14.5 \%$, respectively, at the end of a 7-year follow-up [16]. The annual incidence of heart failure and MD were $0.02 \%$ and $0.1 \%$, respectively. Diastolic heart failure accounted for $85 \%$ of the cases of heart failure $[1,16]$.

\section{PATHOPHYSIOLOGICAL MECHANISMS OF DIABETIC CARDIOMYOPATHY}

The pathophysiological mechanisms of DCM have not yet been sufficiently elucidated. The occurrence of DCM is multifactorial, and there are various proposed mechanisms including insulin resistance, microvascular impairment, subcellular component abnormalities, metabolic disturbances, cardiac autonomic dysfunction, alterations in the renin-angiotensin-aldosterone system (RAAS), and maladaptive immune responses (Fig. 1) [1,9,17].

A long-standing hypothesis is that hyperglycemia plays a pivotal role in the development of DCM, although multiple complex mechanisms and the interplay of many metabolic and molecular events within the

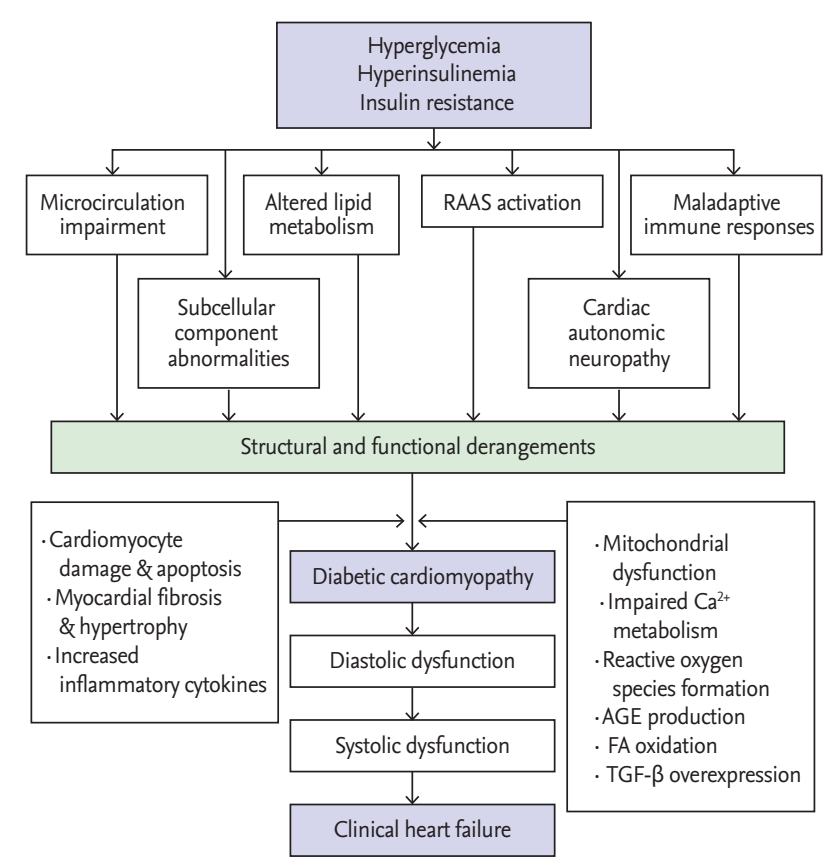

Figure 1. Pathophysiological mechanisms of diabetic cardiomyopathy. RAAS, renin-angiotensin-aldosterone system; AGE, advanced glycation end product; FA, fatty acid; TGF- $\beta$, transforming growth factor- $\beta$. 
myocardium and plasma contribute to its pathogenesis. The main metabolic abnormalities in diabetes mellitus are hyperglycemia, inflammation, and hyperlipidemia, all of which induce the production of the nitrogen species or reactive oxygen species (ROS) that cause most diabetic complications, including DCM and diabetic nephropathy [18].

\section{Effects of hyperglycemia on the heart}

Persistent hyperglycemia causes a number of molecular and metabolic changes in cardiomyocytes. Increased glucose metabolism due to hyperglycemia increases oxidative stress via the development of ROS from the mitochondria [19]. Oxidative stress caused by the overproduction of superoxide in the mitochondrial respiratory chain leads to reduced myocardial contractility and eventually induces myocardial fibrosis [20]. Oxidative stress and ROS can accelerate cardiomyocyte apoptosis and cellular DNA damage. Oxidative stress-induced DNA damage also activates DNA reparative enzymes such as poly ADP ribose polymerase (PARP) [21]. PARP redirects glucose metabolism from its usual glycolytic pathway to an alternative biochemical pathway that results in the development of various mediators and causes hyperglycemia-induced cellular injury. These injuries include increased hexosamine and polyol flux, protein kinase $C$ activation, and advanced glycation end product (AGE) levels. The oxidative stress induced by persistent hyperglycemia increases the amount of AGEs in diabetic subjects [20]. AGEs can covalently crosslink various extra- and intracellular proteins that are thought to be important factors in diabetic complications. The crosslinking involving elastin and collagen results in impaired cardiac relaxation and increased myocardial stiffness. AGEs cause myocardial damage in both humans [22] and animals [23].

\section{Contributions of insulin resistance and hyperinsu- linemia}

Insulin resistance and hyperinsulinemia are typical pathological abnormalities in T2DM and prediabetic conditions. Hyperinsulinemia leads to cardiomyocyte hypertrophy via various mechanisms. Cardiomyocyte hypertrophy in diabetes mellitus is modulated at the transcriptional level [24]. Various epigenetic and genetic alterations resulting from hyperinsulinemia activate multiple transcription factors that regulate extracellular and cellular protein expression. The activation of such transcription factors causes the deposition of extracellular matrix proteins and cardiomyocyte hypertrophy, resulting in focal myocardial fibrosis in diabetes mellitus [24].

\section{Microcirculation impairment in the myocardium}

The pathological characteristic of diabetes-related vascular complications is damage to the microcirculation throughout the body. Unique examples of microvascular complications inlcude diabetic nephropathy, neuropathy, and retinopathy [1].

Impaired coronary microvasculature is frequently observed in patients with $\mathrm{T}_{2} \mathrm{DM}$, insulin resistance, and DCM $[25,26]$. This defect is caused by reduced levels of bioavailable nitric oxide [27]. In coronary vascular smooth muscle cells, nitric oxide activates both kinases and guanylyl cyclase, which is required for coronary relaxation [28]. Under conditions of reduced insulin sensitivity, both increased nitric oxide degradation and reduced nitric oxide production occur.

Reduced capillary length density and hyaline-related changes in the medial arteriolar layers are observed in the cardiac circulation of diabetes mellitus patients $[17,29]$. The reduced blood supply resulting from microcirculatory dysfunction affecting the vasa vasorum in diabetes mellitus further damages the medium and small arterioles of the diabetic heart. Perivascular fibrosis and interstitial changes, the formation of microaneurysms in small vessels, and thickening of the capillary basement membrane are other vascular disorders that cause cardiac microvascular ischemia in diabetes mellitus. Ischemia contributes to myocardial fibrosis, stiffness, and dysfunction in DCM.

Hyperinsulinemia and insulin resistance are associated with stiffness of both the small and large blood vessels [30]. Hyperinsulinemia promotes the differentiation of vascular smooth muscle cells to an osteoblast-like phenotype, which could play a role in the observed increased vascular stiffness [31]. Elevated insulin levels might also enhance vascular stiffness by increasing osteocalcin expression, alkaline phosphatase activity, and the formation of mineralized nodules in vascular smooth muscle cells via increased levels of receptor activator of nuclear factor $\kappa \mathrm{B}$ [32]. Thus, impaired vascular 
smooth muscle cell and endothelial cell function are related to an increased risk of developing CAD in association with DCM.

\section{Subcellular component abnormalities}

Metabolic abnormalities involving endoplasmic reticulum (ER) stress, impaired calcium handling, and mitochondrial dysfunction are associated with the pathogenesis of DCM [11,33,34].

The overproduction of ROS exerts deleterious effects on the ER by impairing post-translational modifications and protein folding in the rough ER [25]. Stress to the ER induces an adaptive response that increases the proteasomeal degradation of incorrectly folded proteins [34].

Under conditions of excessive fat and carbohydrate intake and insulin resistance, nutrient overflow into cells causes the transfer of electrons to oxygen without adenosine triphosphate (ATP) production and also promotes a state of increased ROS, which potentially leads to oxidative damage within mitochondria [35]. Therefore, in the presence of mitochondria-produced ROS, DNA, proteins, and lipid membrane components are damaged and the accumulation of ROS-mediated fibrosis promotes diastolic dysfunction that can progress to heart failure.

ER and oxidative stress can impair calcium handling, which subsequently causes diastolic dysfunction and DCM. ROS, long-chain acylcarnitines, and abnormal membrane lipid content in the mitochondrial membrane, such as via cardiolipin, can alter calcium handling by affecting various transporter proteins; thus, inducing delayed diastolic relaxation and impaired intracellular calcium uptake $[17,36]$. The interactions between abnormal calcium handling, ROS, and ER stress enhance subcellular component dysfunction and ultimately lead to autophagy, necrosis, and apoptosis [26,37].

\section{Effects of altered lipid metabolism on the myocar- dium}

The heart can use both glucose and fatty acids (FAs) as energy substrates under physiological conditions. The uptake of FAs is mediated by FA translocase and the cluster of differentiation $36\left(\mathrm{CD}_{3} 6\right)$, whereas glucose intake occurs via insulin-stimulated glucose transport, which is mediated by glucose transporter 4 (GLUT4) $[11,38]$. Nutrients promote myocardial insulin signaling and increase plasma insulin levels, which enhance the translocation of GLUT 4 and $\mathrm{CD}_{3} 6$ to the myocyte sarcolemma to supply myocardial energy substrates [11]. In contrast, under conditions of T2DM and/or insulin resistance, $\mathrm{GLUT}_{4}$ is internalized and returns to its intracellular location and $\mathrm{CD}_{3} 6$ becomes preferentially localized to the sarcolemma [39].

The reciprocal positioning of $\mathrm{CD}_{3} 6$ and GLUT4 influences the genesis of cardiac metabolic abnormalities that are characterized by metabolic inflexibility [39]. Thus, the reduced glucose uptake caused by cardiac and systemic insulin resistance promotes a substrate shift toward increased free fatty acid (FFA) oxidation in diabetes mellitus, resulting in reduced cardiac efficiency $[33,40]$. The lipotoxicity characterized by the excessive accumulation of FAs in the myocardium reduces normal physiological autophagy and impairs insulin signaling, which leads to structural and morphological alterations and impaired myocardial performance [33]. These abnormalities facilitate myocardial oxygen consumption and can reduce the efficiency of muscle fiber function in response to electrical stimuli $[17,36]$.

Overstrain of the cellular oxidation capacity in diabetes mellitus leads to ectopic lipid deposition in non-adipose tissues such as the heart, liver, and skeletal muscle. Recently, cardiac steatosis was proposed as an important cause of DCM $[41,42]$. The contribution of glucose oxidation to cardiac energetics is less than normal among patients with T2DM and obesity, and FA metabolism, instead, meets the myocardial energy needs [43]. Increased plasma FFA levels in patients with obesity and T2DM cause increased cardiac triglyceride accumulation and FA uptake. Excessive FA uptake and delivery by cardiomyocytes in this setting is likely to exceed the mitochondrial oxidative capacity and, thus, induces lipotoxic injury to the myocardium. Some of the excess FA enter nonoxidative pathways, leading to toxic FA intermediates such as ceramide. These toxic substances subsequently interfere with normal cellular signaling and give rise to apoptosis, cellular damage, mitochondrial dysfunction and, eventually, contractile dysfunction and myocardial fibrosis. Increased FA oxidation in the mitochondria is associated with the increased production of ROS, which oxidize cytoplasmic lipids into lipid peroxides. In turn, lipid peroxides and ROS lead to mitochondrial and cellular damage and the uncoupling of 
mitochondrial oxidative metabolism [44]. Consequently, the impaired myocardial generation of energy and reduced cardiac contractility are observed. Decreased energy production also induces impaired mitochondrial calcium handling, which leads to cardiac dysfunction [45]. Lipotoxicity-induced cellular apoptosis is generally referred to as lipoapoptosis. Different mechanisms may induce lipoapoptosis, such as palmitate toxicity, ER stress, diacylglycerol and ceramide formation, inflammation, and membrane destabilization [44]. The effects of lipoapoptosis on cardiac function include myocardial fibrosis and structural damage.

\section{Cardiac autonomic neuropathy in diabetic cardiomy- opathy}

A previous study reported a relationship between the development of DCM and nervous system activation states [46]. Activation of the sympathetic nervous system increases $\beta 1$-adrenergic signaling and expression, which facilitates interstitial fibrosis, cardiomyocyte hypertrophy, and impaired contractile function accompanied by increased cardiomyocyte apoptosis [17,36]. Activation of the parasympathetic nervous system is reduced, muscarinic receptor composition and density are altered, and acetylcholinesterase activity is decreased during heart failure [47]. Either indirect or direct stimulation of the vagus nerve might have direct beneficial effects on both cardiac remodeling and clinical outcomes [47].

CAN is a chronic complication of diabetes mellitus that leads to abnormalities in vascular hemodynamics and heart rhythm. The prevalence of varying degrees of CAN may be as high as $60 \%$ in individuals with a persistent history of diabetes mellitus [48]. CAN changes the contractile function of the myocardium and also influences blood flow in the coronary circulation. Patients with CAN exhibit increased peripheral vascular resistance and reduced vascular elasticity due to abnormal sympathetic tone [49]. A reduction in the myocardial perfusion reserve was also reported by other researchers [50]. This may partially account for the ventricular dysfunction associated with diabetic CAN.

Cardiac dysfunction is usual in diabetic patients with CAN [51]. Correlations between the prevalence of diastolic dysfunction and the severity of CAN have also been reported [51]. Alterations in the myocardial contractility responses associated with stress are observed in patients with diabetic CAN, and exercise-induced MD has been recorded even in patients with normal ventricular function at rest [52].

\section{Activation of the renin-angiotensin-aldosterone system}

Current evidence from human and animal experiments has supported a significant role for RAAS in diabetes-induced cardiac dysfunction [53,54]. Hyperglycemia activates the intracardiac RAAS, which exhibits different effects on cardiomyocytes. Intracellular angiotensin (AGT) II levels were 3.4-fold higher in the myocardial cells of diabetic patients compared with non-diabetics [55]. Cytoplasmic AGT II promotes cell growth in animal models. AGT II has a direct effect on cell signaling, leading to the proliferation of cardiac fibroblasts and cardiomyocyte hypertrophy [53]. Other factors, such as inflammation, aldosterone, and oxidative stress, may lessen the harmful effects of AGT II on the heart that cause myocardial damage in diabetes mellitus [54]. Moreover, the enhanced activation of mineralocorticoid receptor signaling and AGT II might facilitate insulin resistance by activating the mammalian target of rapamycin-S6 kinase 1 signal transduction pathway [56].

\section{Maladaptive immune responses}

DCM can be facilitated by alterations in the adaptive and innate immune systems [57,58]. The activation of macrophage polarization to classic (M1) or alternative (M2) phenotypes and proinflammatory $\mathrm{T}$ helper cells often occurs in states of insulin resistance or obesity [59]. Moreover, persistent overfeeding induces immune responses that contribute to low-grade inflammation within white adipose tissue [38]. Macrophage M2 polarization is an anti-inflammatory response and Mi polarization is a proinflammatory response in insulin-resistant and obesity states [59,60]. M1 macrophages secrete inflammatory cytokines, which reduce cardiac and systemic insulin signaling and facilitate the development of DCM [59]. In contrast, M2 macrophages secrete macrophage mannose receptor 1 and interleukin 10, which reduce the development of myocardial fibrosis and cardiomyocyte hypertrophy [59,61].

Another population of immune cells ( $T$ helper lymphocytes) was identified in patients with DCM [62]. A higher $\mathrm{CD}^{+}: \mathrm{CD}_{4}{ }^{+} \mathrm{T}$-cell ratio is found in the visceral 
adipose tissue of mice fed a high-fat diet compared with lean mice $[63,64]$. Diet-induced insulin resistance also induces a dramatic increase in type $1 \mathrm{~T}$ helper-polarized cells, whereas the type $2 \mathrm{~T}$ helper-polarized fraction is reduced by $\sim 50 \%$ [63]. These cells can be subtyped according to their cytokine expression profile following activation [64]. The increased secretion of chemokines, growth factors, and proinflammatory cytokines by $\mathrm{T}$ helper lymphocytes results in increased impaired diastolic relaxation and cardiac fibrosis [65]. However, regulatory T-cells usually attenuate the proinflammatory effects of T helper cells in the heart [66].

\section{FUNCTIONAL AND STRUCTURAL ALTERATIONS}

Significant alterations in myocardial function and structure occur as a result of DCM, leading to the pathological-clinical consequences of the disease. Pathological changes occur mostly in the myocardial interstitium in the early stages of the disease, and cardiac contractile dysfunction ensues as a consequence of these alterations [67]. Later, cardiac microcirculatory dysfunction, interstitial and perivascular fibrosis, and ventricular myocardial hypertrophy are observed.

Impaired diastolic function is the earliest abnormality in DCM, and systolic dysfunction develops only in the later stages of the disease $[67,68]$. DCM-induced ventricular fibrosis and hypertrophy are the major causes of diastolic dysfunction. When systolic dysfunction occurs, cardiac output declines progressively with the severity of the disease.

\section{Interaction with coexistent coronary artery disease and hypertension}

DCM is diagnosed only when CAD and hypertension have been ruled out. However, when these diseases overlap with existing DCM, the rapid progression to advanced heart failure may ensue. It is difficult to verify the role of these diseases in the progression and development of DCM from a clinical perspective [1].

Coexistent hypertension was observed in nearly 30\% of patients with TiDM and in $50 \%$ to $80 \%$ of patients with T2DM in the United States [69]. Cardiac dysfunction was reportedly worsened by hypertension in animal models of DCM [70]. The presence of hypertension was independently associated with diastolic dysfunction in diabetic patients [71].

\section{Cardiac remodeling in diabetic cardiomyopathy}

DCM originates from the functional, structural, and regulatory remodeling of the heart caused by diabetes mellitus. Different stages of remodeling have been proposed, such as the early, advanced, and late stages [72].

In the early stage of DCM, metabolic disturbances such as hyperglycemia and insulin resistance are not accompanied by substantial alterations in myocardial systolic function and structure $[36,37]$. However, impaired myocardial relaxation can be detected by magnetic resonance imaging (MRI) and echocardiography. DCM is initially characterized by increased impairments in relaxation and cardiac stiffness with elevated atrial filling and reduced early diastolic filling [36]. Impaired insulin signaling also leads to a decrease in myocardial bloodflow reserve, which can be identified using various imaging techniques [73].

In the advanced stage of DCM, many alterations at the cellular level increase cardiac fibrosis, which induces substantial changes in systolic and diastolic function [17].

In the late stage of DCM, changes in neurohumoral activation, metabolism, and the development of myocardial fibrosis lead to further deterioration in coronary microcirculation and systolic and diastolic function [37,74].

\section{DIAGNOSIS OF DIABETIC CARDIOMYOPATHY}

A number of DCM cases are subclinical, and patients may not have any overt signs or symptoms of the disease. In the early stages, there are only substructural cardiomyocyte changes, and detection is possible only using very sensitive methods such as strain rate, strain, and myocardial tissue velocity [17]. Subsequently, myocardial fibrosis and hypertrophy develop, which may be associated with structural changes such as increased myocardial mass and LV hypertrophy. Conventional diagnostic methods such as echocardiography may detect systolic and/or diastolic dysfunction at this stage. Significant fibrosis and microcirculatory changes occur in the myocardium in the advanced stages of DCM; this stage is usually associated with overt heart failure, ischemic 
Table 1. Diagnostic modalities for diabetic cardiomyopathy

\begin{tabular}{|c|c|c|}
\hline Tool & Assessment & Parameter \\
\hline \multirow[t]{3}{*}{ Echocardiography } & Structural changes & 2D for LV hypertrophy \\
\hline & Functional changes & Mitral inflow for diastolic function \\
\hline & & TDI for diastolic and systolic function \\
\hline \multirow[t]{3}{*}{ Cardiac MRI } & Structural changes & LV hypertrophy, myocardial steatosis \\
\hline & Functional changes & $\begin{array}{l}\text { Late gadolium-enhancement for diastolic and systolic } \\
\text { function }\end{array}$ \\
\hline & Metabolic changes & MRS for myocardial TG content and PCr/ATP \\
\hline Cardiac PET & Metabolic and hemodynamic changes & Myocardial metabolic abnormality and blood flow \\
\hline Coronary angiography & Functional and hemodynamic changes & $\begin{array}{l}\text { Mean PCWP and LVEDP for diastolic function, } \\
\text { microvascular CAD }\end{array}$ \\
\hline \multirow[t]{5}{*}{ Serology } & Structural changes & MMPs and TIMPs for myocardial fibrosis \\
\hline & Functional changes & mi-RNA for contractile function \\
\hline & & $\mathrm{P}_{3} \mathrm{NP}$ for LV dysfunction \\
\hline & & BNP for LV diastolic and systolic function \\
\hline & & Troponin for LV dysfunction \\
\hline
\end{tabular}

2D, two-dimensional echocardiography; LV, left ventricular; TDI, tissue Doppler imaging; MRI, magnetic resonance imaging; MRS, magnetic resonance spectroscopy; TG, triglyceride; PCr, phosphocreatine; ATP, adenosine triphosphate; PET, positron emission tomography; PCWP, pulmonary capillary wedge pressure; LVEDP, left ventricular end-diastolic pressure; CAD, coronary artery disease; MMP, matrix metalloproteinase; TIMP, tissue inhibitor of MMP; mi-RNA, micro-ribonuleic acid; $\mathrm{P}_{3} \mathrm{NP}$, procollagen $3 \mathrm{~N}$-terminal peptide; BNP, brain natriuretic peptide.

heart disease, and hypertension [17]. Recently, several biomarkers have emerged for the detection of metabolic changes (Table 1).

\section{Echocardiography}

Echocardiography is a relatively inexpensive diagnostic method for assessing functional and structural cardiac abnormalities. Transmitral Doppler is the most commonn technique for evaluating LV diastolic function [75]. Tissue Doppler imaging (TDI) measures myocardial tissue velocities during the cardiac cycle and can be used to quantitatively estimate regional and global diastolic and systolic myocardial functions [76]. TDI is a more sensitive and specific diagnostic tool for detecting DCM compared with transmitral Doppler [77]. Fortunately, newer echocardiographic imaging techniques are currently evolving with better sensitivities and specificities.

\section{Magnetic resonance imaging}

Cardiac MRI recently emerged as a well-accepted imaging tool for the diagnosis of various functional and structural myocardial disorders [78]. Cardiac MRI is also useful for identifying myocardial steatosis and diastolic dysfunction [42]. Positron emission tomography (PET) and cardiac MRI using different radionuclides can help assess myocardial metabolic abnormalities; these newer imaging techniques may be helpful for diagnosing DCM.

\section{Coronary angiography and cardiac catheterization}

Cardiac catheterization is the best tool for evaluating the hemodynamic events within the heart chambers, since diastolic dysfunction documented invasively through catheterization continues to be the most definitive evidence of diastolic heart failure [79]. A mean pulmonary capillary wedge pressure $>12 \mathrm{mmHg}$ or an LV end-diastolic pressure $>16 \mathrm{mmHg}$, determined invasively by catheterization, are the best diagnostic features of diastolic dysfunction [79]. However, the catheter-based diagnosis of DCM is rarely used because of the availability of highly sensitive and specific noninvasive techniques. Coronary angiography is helpful for detecting CAD that may coexist with or complicate DCM. Microvascular $\mathrm{CAD}$ is also detected by coronary angiography.

\section{Serologic markers}

Alterations in the levels of various serum/plasma cardi- 
ac biomarkers may reveal some myocardial structural and metabolic functions. A strong correlation between ongoing cardiac remodeling and the turnover of extracellular matrix proteins was reported in different studies $[80,81]$. Matrix metalloproteinases (MMPs) are the enzymes that degrade the extracellular matrix, increase matrix turnover, and change the expression of several micro-ribonucleic acids (mi-RNAs) that induce contractile dysfunction of the myocardium [72]. Elevated levels of MMPs, especially MMP-9, and reduced levels of the tissue inhibitors of MMPs are observed in myocardial fibrosis. The clinical usefulness of these new biomolecules for the diagnosis of DCM is currently under investigation.

Serum aminoterminal propeptide of type III, an indicator of type III collagen turnover in the body, was proposed as an early index of LV dysfunction in obese subjects with insulin resistance [82].

Recently, brain natriuretic peptide (BNP) emerged as a helpful biomarker for screening subclinical ventricular diastolic dysfunction in patients with uncontrolled diabetes $[83,84]$. Epshteyn et al. [85] showed a high positive predictive value of $96 \%$ for plasma BNP levels $(>90 \mathrm{pg} / \mathrm{mL})$ in diabetic patients for the diagnosis of LV dysfunction with echocardiographic correlation.

Cardiac troponins ( $\mathrm{T}, \mathrm{N}$, and I) are biomarkers released into circulation from the injured myocardium in inflammatory or ischemic disease. Elevated troponin $\mathrm{T}$ levels were found in infants with cardiac dysfunction and cardiomyopathy born to diabetic mothers [86]. However, the role of troponins for the assessment of adult patients with DCM is unclear.

Mi-RNAs are small non-coding RNA molecules that coordinate cellular gene expression. The dysregulation of mi-RNAs has been linked to diabetes and many of its complications. Altered levels of mi-RNAs were observed in the cardiomyocytes of experimental diabetic models [87]. These novel biomarkers may emerge as prognostic and diagnostic methods for patients with DCM in the future.

\section{Detection of metabolic changes}

A reduction in the phosphocreatine (PCr)/ATP ratio suggests suppressed ATP production and/or the suppressed production of PCr from ATP by the creatine kinase system. Determining PCr and ATP levels in the hu- man myocardium by magnetic resonance spectroscopy (MRS) demonstrated that the PCr/ATP ratio is significantly reduced in diabetes and that the ratio is negatively correlated with plasma FFA levels or live triglyceride levels [77,88]. A promising novel approach for the diagnosis of DCM is the characterization of metabolic changes in the myocardium using ${ }^{31} \mathrm{P}-\mathrm{MRS}$ and ${ }^{1} \mathrm{H}-\mathrm{MRS}$. The $\mathrm{PCr} /$ ATP ratio, an indicator of energy charge, is reduced in the myocardium of diabetic subjects compared with control patients. Recent studies using ${ }^{1} \mathrm{H}$-MRS showed that an increase in myocardial triglyceride content was associated with LV diastolic dysfunction in diabetic subjects $[41,42]$.

\section{PREVENTION AND THERAPEUTIC STRATEGIES}

Recently, a better understanding of the pathophysiology and pathogenesis in patients with DCM has provided improved management options. These include lifestyle modifications, improved diabetic control, the management of coexistent hypertension and CAD if present, lipid-lowering therapies, and the management of heart failure (Table 2) [1].

\section{Lifestyle modifications}

Weight loss, limitation of fat and total energy intake, and regular physical activity can positively adjust metabolic abnormalities and improve tissue and systemic insulin resistance by increasing insulin-mediated glucose transport and post-receptor insulin signaling, which seems to be related to facilitated signal transduction at the level of phosphatidylinositol 3-kinase and insulin receptor substrate $[56,67]$.

Physical activity was associated with a significant reduction in cardiovascular disease and all-cause mortality in patients with diabetes mellitus in many clinical studies [89]. Exercise training was beneficial for reducing the incidence of DCM in both human patients and animal models $[83,84]$. It is difficult to predict the benefit of physical activity in documented cases of the disease in the absence of controlled clinical trials. However, better diabetic control with regular exercise would have beneficial effects on disease outcome. Maintaining healthy eating patterns that are suitable for diabetic subjects can also be expected to offer similar beneficial effects [1]. 
Table 2. Therapeutic strategies or targets for diabetic cardiomyopathy

\begin{tabular}{ll}
\hline Modality & \multicolumn{1}{c}{ Implication } \\
\hline Lifestyle modification & Improve insulin resistance, reduction of CVD and all-cause mortality \\
Anti-diabetic medications & Metformin: upregulate cardiac autophagy, reduce mortality \\
& TZD: improve cardiac dysfunction and myocardial glucose uptake \\
& GLP-1: attenuate myocardial apoptosis, enhance vasodilation \\
& DPP-4 inhibitor: prevent cardiac diastolic dysfunction by inhibition of fibrosis and oxidative \\
& stress \\
& Empagliflozin: control visceral adiposity, BP, arterial stiffness, albuminuria, weight, \\
& oxidative stress, hyperinsulinemia, and uric acid level \\
Vasoactive medications & ACEi/ARB: improve HF symptom and reduce mortality \\
& BB: reduce hospitalization and mortality, improve HF symptom \\
& PDE-5 inhibitor: improve myocardial function and cardiac remodeling \\
\hline Lipid lowering medications & Statin: reduce myocardial fibrosis and inflammation, improve LV function \\
Metabolic modulators & Trimetazidine: reduce free radical injury, improve endothelial function, inhibit apoptosis, \\
& attenuate lipotoxicity \\
& Ranolazine: normalize altered cardiomyocyte intracellular calcium concentration \\
\hline
\end{tabular}

CVD, cardiovascular disease; TZD, thiazolidinedione; GLP-1, glucagon-like peptide 1; DPP-4, dipeptidyl peptidase 4; BP, blood pressure; ACEi, angiotensin converting enzyme inhibitor; ARB, angiotensin II receptor blocker; HF, heart failure; BB, $\beta$-blocker; PDE-5, phosphodiesterase 5; LV, left ventricular.

\section{Antidiabetic medications}

Better glycemic control was associated with better outcomes in diabetic microcirculatory complications in many clinical trials. However, the beneficial effects of tight glycemic control on macrovascular outcomes are still unclear. Because microvascular disease plays an important pathogenic role in the development of DCM, rigid glycemic control would be expected to benefit patients [1].

Improved glycemic control delayed DCM in animal models [9o]. Strict glycemic control improved stress-induced ventricular dysfunction without CAD in poorly controlled diabetic patients in a large prospective study [91]. Another case-controlled study using cardiac MRI in patients with $\mathrm{T} 1 \mathrm{DM}$ demonstrated that rigorous glycemic control was associated with better DCM outcome parameters [92]. Diabetes management was also beneficial for reducing myocardial steatosis [93].

Metformin upregulates cardiac autophagy, which plays a role in the prevention of DCM in animal models [94]. Metformin was reported to reduce mortality and improve the clinical outcome in overweight patients with heart failure and diabetes mellitus, in spite of the in- creased risk of lactic acidosis [95]. This drug facilitates glucose uptake and GLUT4 translocation in insulin-resistant cardiomyocytes and the myocardium by activating $5^{\prime}$ adenosine monophosphate-activated protein kinase $[95,96]$. However, there are no data regarding its role in humans with DCM.

Thiazolidinediones, which are insulin sensitizers, improve contractile dysfunction and myocardial glucose uptake by activating PPAR- $\gamma$ [97]. However, thiazolidinedione therapy can cause chronic symptoms that resemble heart failure by increasing water and sodium reabsorption in the kidney collecting tubules and vascular permeability, which induces generalized edema [98]. Therefore, the drug is generally not recommended in patients with heart failure. However, pioglitazone had anti-inflammatory effects that improved myocardial fibrosis in animal models, and so the drug may prevent the development of DCM [99].

Natural glucagon-like peptide 1 (GLP-1) has a very short biological half-life. Synthetic GLP-1 mimetic agents with longer half-lives, such as exenatide and liraglutide, are new anti-diabetic agents being widely used. Their use in obese T2DM patients is associated with significant improvements in weight loss and glycemic control. GLP- 
1 agonists also attenuate cardiomyocyte apoptosis in rat models [100]. GLP-1 encourages insulin secretion, enhances nitric oxide-induced vasodilation, and facilitates glucose use in the myocardium $[97,101]$. This novel group of drugs may emerge as a promising management strategy in obese T2DM patients with DCM.

Dipeptidyl peptidase 4 (DPP- 4 ) is an enzyme that metabolizes endogenous GLP-1. DPP-4 inhibitors prolong the effects of endogenous GLP-1. Agents in this class, such as sitagliptin, linagliptin, saxagliptin, and vildagliptin, are effective anti-diabetic medications. They are weight-neutral and are especially useful in overweight and obese diabetic patients in combination with conventional anti-diabetic agents such as metformin. The use of sitagliptin promotes myocardial glucose uptake in patients with nonischemic cardiomyopathy [102]. DPP-4 inhibitors can prevent cardiac diastolic dysfunction and cardiac hypertrophy by inhibiting fibrosis and oxidative stress in mouse models of insulin resistance and obesity [103]. The possible therapeutic role of DPP-4 inhibitors in patients with DCM is yet to be elucidated.

Amylin analogues reduce $\mathrm{HbAlc}$ values, body weight, and even the insulin requirement when administered together with insulin [104]. Their role in controlling hyperglycemia may benefit patients with DCM.

Empagliflozin, an inhibitor of sodium-glucose cotransporter 2, is a new antidiabetic agent that reduces $\mathrm{HbAic}$ levels in patients with T2DM by controlling visceral adiposity, blood pressure, arterial stiffness, albuminuria, weight, oxidative stress, hyperinsulinemia, and circulating uric acid levels [105]. In the Empagliflozin Cardiovascular Outcome Event Trial in Type 2 Diabetes Mellitus Patients(EMPA-REG OUTCOME)involving patients with T2DM, empagliflozin with standard therapy lowered the rate of primary composite cardiovascular outcome and death compared with placebo added to standard therapy; however, an increased rate of genital infections was noted [106].

\section{Vasoactive medications and coronary intervention}

There are no formal guidelines regarding the management of coexistent cardiac ischemia and hypertension in patients with DCM. However, when these diseases coexist they promote the progression of DCM because of their harmful effects on ventricular structure and function. The optimal treatment of CAD and hypertension would be expected to improve the disease progression and even slow it down. Coronary intervention in appropriate cases with significant CAD may improve the clinical outcomes and symptoms. The management of heart failure depends on the severity, type (diastolic or systolic), and associated conditions like CAD and hypertension [1].

Various vasoactive medications have been tried in both human patients and animal models with DCM, with variable results. The most-studied medications were those where subjects were active on the renin-angiotensin systems. The production of AGT II within the myocardium was proposed as a mechanism for the development of DCM. Recently, angiotensin converting enzyme inhibitor (ACEi), renin inhibitor (aliskiren), and angiotensin II receptor blocker (ARB) were all shown to be protective against DCM in rat models [107]. ARBs and ACEis were also beneficial in both human and animal models of DCM $[108,109]$.

$\beta$-Adrenoreceptor blockers were effective in experimental models of DCM [110]. Because of the proven benefits of $\beta$-blockers in chronic heart failure, this group of drugs should be considered for treatment of DCM; however, there are no reported randomized clinical trials examining the benefits. They can be used as effective antihypertensive agents in DCM patients with hypertension.

Similarly, calcium channel antagonists were beneficial in animal models of DCM [111]. However, data on human subjects are lacking to make evidence-based recommendations for the use of these agents in the management of DCM, especially in the absence of coexistent hypertension.

Sildenafil, a selective phosphodiesterase type 5 inhibitor, was recently shown to improve myocardial function, cardiac remodeling, and some circulatory markers of cardiac inflammation in patients with DCM [112]. Larger clinical trials in the future may determine if this novel agent can be recommended for routine use in patients.

\section{Lipid lowering medications}

Dyslipidemias are more detrimental in diabetic than non-diabetic individuals because of their higher atherogenic potential. The particle size of low density lipoprotein cholesterol (LDL-C) is smaller in diabetic subjects, which is more atherogenic even with near-normal 
plasma levels. The use of statins reduces cardiovascular mortality and events in patients with diabetes and vascular risk factors in multiple clinical trials [113], and is beneficial even for primary prevention in patients without established cardiovascular disease [114]. The vascular remodeling capacity of statins is referred to as the pleiotropic effect.

Atorvastatin, reduces myocardial fibrosis, intramyocardial inflammation, and improves LV function in rat models of experimental DCM, independently of its LDL-C-lowering capacity [115]. Similarly, fluvastatin is useful for attenuating cardiac dysfunction and myocardial interstitial fibrosis in rat models of the disease [116]. Although there are no clinical trials investigating the role of lipid-lowering therapy in individuals with established DCM, the benefits of dyslipidemia treatment can be anticipated in these patients, along with a role in primary disease prevention.

\section{Metabolic modulators}

Trimetazidine, an atypical anti-anginal agent with antioxidant properties, is a competitive inhibitor of the terminal enzyme in $\beta$-oxidation; it might be used to improve substrate or myocardial metabolic flexibility and cardiac function in the setting of DCM [117]. Trimetazidine might affect myocardial substrate use by inhibiting oxidative phosphorylation and shifting energy production from FFAs to glucose oxidation [118]. It might also help reduce the injury caused by free radicals and calcium overload, preserve intracellular ATP and PCr levels, improve endothelial function, and inhibit cellular apoptosis [118]. The drug has promising beneficial effects on heart failure in diabetic patients with both idiopathic and ischemic dilated cardiomyopathy [119]. Animal models revealed that trimetazidine improved myocardial function by augmenting the oxidation status and attenuating lipotoxicity in the heart; therefore, it might suppress the development of DCM [120]. Human trials are needed to investigate the beneficial effects of this well-tolerated drug on the treatment and prevention of DCM.

Ranolazine, a potent late $\mathrm{Na}^{+}$current inhibitor, might normalize altered cardiomyocyte intracellular $\mathrm{Ca}^{2+}$ concentrations due to the close relationship between $\mathrm{Ca}^{2+}$ and $\mathrm{Na}^{+}$handling by the $\mathrm{Na}^{+} / \mathrm{Ca}^{2+}$ exchanger [121]. Ranolazine improved measures of hemodynamics, but not cardi- ac relaxation parameters [121]. A single treatment with ranolazine is probably not sufficient to affect cardiac function and structure [121].

Perhexiline is an inhibitor of carnitine o-palmitoyltransferase 1 ; it increases LV ejection fraction, maximal $\mathrm{O}_{2}$ consumption $\left(\mathrm{VO}_{2}\right.$ max), resting and peak stress myocardial function, and skeletal muscle energetics [117]. In patients with chronic heart failure, a multicenter double-blind randomized controlled trial showed that perhexiline improved LV ejection fraction, resting and peak stress myocardial function, $\mathrm{VO}_{2}$ max, and skeletal muscle energetics. Despite these benefits, the use of the perhexiline is diminishing due to reports of peripheral neuropathy and hepatotoxicity [122].

Although experimental models have shown that $\alpha$-lipoic acid suppresses cardiac fibrosis and has beneficial effects on cardiac redox homeostasis [123], human data are not yet available. Other investigational antioxidants that were useful in recent animal models include resveratrol [124], luteolin [125], riboflavin [126], and sodium ferulate [127].

Resveratrol reduces blood glucose levels and improves cardiac function and insulin sensitivity in patients with heart failure [128]. Although resveratrol is associated with improved triglyceride levels, heart rate, and glycemia, it does not prevent cardiovascular- or cancer-, or all-cause mortality [129].

\section{EMERGING TREATMENT MODALITIES}

\section{Targeting mitochondrial oxidative stress}

Given the importance of oxidative stress in the development of DCM, targeting excess myocardial ROS production with novel antioxidants might offer a promising approach for preventing DCM (Table 3).

The Szeto-Schiller peptide peptide d-Arg-2', 6'-dimethyltyrosine-Lys-Phe-NH2 (SS31) is a positively charged free-radical scavenger that can accumulate to high levels in the mitochondria and prevent diastolic dysfunction, fibrosis, and cardiac hypertrophy [101,130]. SS31 prevents cardiolipin from converting cytochrome into a peroxidase while protecting the cardiolipin electron-carrying function by interacting with cardiolipin [131]. SS31 also protects the structure of mitochondrial cristae and promotes oxidative phosphorylation [131]. Thus, SS31 rep- 
Table 3. Emerging treatment modalities or targets

\begin{tabular}{ll}
\hline Modality & \multicolumn{1}{c}{ Implication } \\
\hline Emerging agents or targets & $\begin{array}{l}\text { SS31: prevent diastolic function, fibrosis, and cardiac hypertrophy, promote oxidative } \\
\text { phosphorylation }\end{array}$ \\
& Coenzyme Q10: improve HF symptom, reduce all-cause mortality and cardiovascular death \\
& Pim-1 gene: improve LV diastolic function, prevent cardiac apoptosis, fibrosis, and \\
development of HF & mi-RNA: potential biomarker for early detection of DCM \\
\hline
\end{tabular}

SS31, peptide d-Arg-2', 6'-dimethyltyrosine-Lys-Phe-NH2; HF, heart failure; Pim-1, serine/threonine-protein kinase pim-1; LV, left ventricular; mi-RNA, micro-ribonucleic acid; DCM, diabetic cardiomyopathy.

resents a new class of compounds that can restore bioenergetics and recharge the cellular powerhouse.

Coenzyme Q10 improves cardiac function in patients with diabetes mellitus and concurrent heart failure $[130,132]$. A randomized controlled trial showed that long-term coenzyme Q10 treatment in patients with chronic heart failure is safe, improves symptoms, and reduces all-cause mortality by $42 \%$ and cardiovascular death by $43 \%$ [133].

\section{Cell- and genetic-based therapy}

Cell-based therapy and the genetic correction of abnormalities are potential strategies to prevent the development of DCM or treat the early stages of this condition [134].

For example, the gene delivery of nerve growth factor preserves microvessel density, cardiac perfusion, and LV diastolic and systolic function [135]. The systemic administration of Pim-1 improves LV diastolic function and prevents cardiac apoptosis, fibrosis, and the development of heart failure [136].

The dysregulation of mi-RNA function is an important pathogenic mechanism of diabetes and its complications such as DCM, and the artificial restoration of normal function can be a potential therapeutic target. Six mi-RNAs (miR-34b, miR-34c, miR-199b, miR-210, miR223, and miR-650) might be involved in the pathogenesis of the failing myocardium in patients with diabetes mellitus [137]. Thus, mi-RNAs have become an active area of investigation to determine their potential contribution to heart disease in patients with T2DM. Mi-RNA-based therapies might be beneficial in patients with diabetes mellitus and cardiac pathology [137]. Specific mi-RNA targets are useful for the treatment of structural heart disease in mice [138]. Similarly, the transplantation of bone marrow-derived endothelial progenitor cells (stem cell therapy) ameliorates DCM in rat models [139]. Ongoing investigations may help us to translate the success of these experimental models into clinical practice in the future.

\section{CONCLUSIONS}

DCM is an important but lesser-elucidated complication of chronic diabetes, and is associated with significant cardiac mortality and morbidity. The wide discrepancy in the reported prevalence of DCM may be related to disparities in the types of diagnostic test used by various researchers. The pathophysiology and pathogenesis of DCM are still not fully understood, although proposed mechanisms include insulin resistance, microvascular disease, lipotoxicity, chronic hyperglycemia-associated metabolic and oxidative stress, coexistent CAD and hypertension, CAN, and altered immune responses. The manifestations of DCM can vary from subclinical ventricular dysfunction to overt heart failure. Echocardiography is currently the standard clinical diagnostic method for DCM. Newer investigative modalities such as cardiac MRI, PET imaging, radionuclide scans, and various serum/plasma markers are emerging as diagnostic tools. The management of DCM covers lifestyle modifications, medications for heart failure, good glycemic control, and the treatment of coexistent CAD, dyslipidemia, and hypertension. Novel therapeutic approaches including targeting mitochondrial oxidative stress and cell- or gene-based therapy are currently being investigated. Further research is needed to understand the exact mechanisms involved in the development and course of DCM to enhance the discovery of 
clinically effective targets for preventing this condition and its progression to heart failure.

\section{Conflict of interest}

No potential conflict of interest relevant to this article was reported.

\section{Acknowledgments}

This work was supported by grants of the Basic Science Research Program through the National Research Foundation of Korea (NRF) funded by the Korean Government (2016R1A4A1009895 and 2012R1A1A2008177).

\section{REFERENCES}

1. Pappachan JM, Varughese GI, Sriraman R, Arunagirinathan G. Diabetic cardiomyopathy: pathophysiology, diagnostic evaluation and management. World J Diabetes 2013;4:177-189.

2. Wang ZV, Hill JA. Diabetic cardiomyopathy: catabolism driving metabolism. Circulation 2015;131:771-773.

3. Trachanas K, Sideris S, Aggeli C, et al. Diabetic cardiomyopathy: from pathophysiology to treatment. Hellenic J Cardiol 2014;55:411-421.

4. Finck BN, Lehman JJ, Leone TC, et al. The cardiac phenotype induced by PPARalpha overexpression mimics that caused by diabetes mellitus. J Clin Invest 2002;109:121130.

5. Aneja A, Tang WH, Bansilal S, Garcia MJ, Farkouh ME. Diabetic cardiomyopathy: insights into pathogenesis, diagnostic challenges, and therapeutic options. Am J Med 2008;121:748-757.

6. Kannel WB, McGee DL. Diabetes and cardiovascular disease: the Framingham study. JAMA 1979;241:2035-2038.

7. Ryden L, Armstrong PW, Cleland JG, et al. Efficacy and safety of high-dose lisinopril in chronic heart failure patients at high cardiovascular risk, including those with diabetes mellitus: results from the ATLAS trial. Eur Heart J 2000;21:1967-1978.

8. Shindler DM, Kostis JB, Yusuf S, et al. Diabetes mellitus, a predictor of morbidity and mortality in the Studies of Left Ventricular Dysfunction (SOLVD) Trials and Registry. Am J Cardiol 1996;77:1017-1020.

9. Jia G, DeMarco VG, Sowers JR. Insulin resistance and hyperinsulinaemia in diabetic cardiomyopathy. Nat Rev
Endocrinol 2016;12:144-153.

10. Wong AK, AlZadjali MA, Choy AM, Lang CC. Insulin resistance: a potential new target for therapy in patients with heart failure. Cardiovasc Ther 2008;26:203-213.

11. Aroor AR, Mandavia CH, Sowers JR. Insulin resistance and heart failure: molecular mechanisms. Heart Fail Clin 2012;8:609-617.

12. Maisch B, Alter P, Pankuweit S. Diabetic cardiomyopathy: fact or fiction? Herz 2011;36:102-115.

13. Witteles RM, Fowler MB. Insulin-resistant cardiomyopathy clinical evidence, mechanisms, and treatment options. J Am Coll Cardiol 2008;51:93-102.

14. Nicolino A, Longobardi G, Furgi G, et al. Left ventricular diastolic filling in diabetes mellitus with and without hypertension. Am J Hypertens 1995;8:382-389.

15. Redfield MM, Jacobsen SJ, Burnett JC Jr, Mahoney DW, Bailey KR, Rodeheffer RJ. Burden of systolic and diastolic ventricular dysfunction in the community: appreciating the scope of the heart failure epidemic. JAMA 2003;289:194-202.

16. Konduracka E, Cieslik G, Galicka-Latala D, et al. Myocardial dysfunction and chronic heart failure in patients with long-lasting type 1 diabetes: a 7-year prospective cohort study. Acta Diabetol 2013;50:597-606.

17. Fang ZY, Prins JB, Marwick TH. Diabetic cardiomyopathy: evidence, mechanisms, and therapeutic implications. Endocr Rev 2004;25:543-567.

18. Nishikawa T, Edelstein D, Du XL, et al. Normalizing mitochondrial superoxide production blocks three pathways of hyperglycaemic damage. Nature 2000;404:787-790.

19. Cai L, Li W, Wang G, Guo L, Jiang Y, Kang YJ. Hyperglycemia-induced apoptosis in mouse myocardium: mitochondrial cytochrome C-mediated caspase-3 activation pathway. Diabetes 2002;51:1938-1948.

20. Aragno M, Mastrocola R, Medana C, et al. Oxidative stress-dependent impairment of cardiac-specific transcription factors in experimental diabetes. Endocrinology 2006;147:5967-5974.

21. Du X, Matsumura T, Edelstein D, et al. Inhibition of GAPDH activity by poly(ADP-ribose) polymerase activates three major pathways of hyperglycemic damage in endothelial cells. J Clin Invest 2003;112:1049-1057.

22. Gawlowski T, Stratmann B, Stork I, et al. Heat shock protein 27 modification is increased in the human diabetic failing heart. Horm Metab Res 2009;41:594-599.

23. Petrova R, Yamamoto Y, Muraki K, et al. Advanced gly- 
cation endproduct-induced calcium handling impairment in mouse cardiac myocytes. J Mol Cell Cardiol 2002;34:1425-1431.

24. Feng B, Chen S, Chiu J, George B, Chakrabarti S. Regulation of cardiomyocyte hypertrophy in diabetes at the transcriptional level. Am J Physiol Endocrinol Metab 2008;294:E1119-E1126.

25. Factor SM, Minase T, Cho S, Fein F, Capasso JM, Sonnenblick EH. Coronary microvascular abnormalities in the hypertensive-diabetic rat: a primary cause of cardiomyopathy? Am J Pathol 1984;116:9-20.

26. Adameova A, Dhalla NS. Role of microangiopathy in diabetic cardiomyopathy. Heart Fail Rev 2014;19:25-33.

27. Zhou X, Ma L, Habibi J, et al. Nebivolol improves diastolic dysfunction and myocardial remodeling through reductions in oxidative stress in the Zucker obese rat. Hypertension 2010;55:880-888.

28. Hayden MR, Habibi J, Joginpally T, Karuparthi PR, Sowers JR. Ultrastructure study of transgenic Ren2 rat aorta. Part 1: rndothelium and intima. Cardiorenal Med 2012;2:66-82.

29. Campbell DJ, Somaratne JB, Jenkins AJ, et al. Impact of type 2 diabetes and the metabolic syndrome on myocardial structure and microvasculature of men with coronary artery disease. Cardiovasc Diabetol 2011;10:80.

30. Blaha MJ, DeFilippis AP, Rivera JJ, et al. The relationship between insulin resistance and incidence and progression of coronary artery calcification: the Multi-Ethnic Study of Atherosclerosis (MESA). Diabetes Care 2011;34:749-751.

31. Olesen P, Nguyen K, Wogensen L, Ledet T, Rasmussen LM. Calcification of human vascular smooth muscle cells: associations with osteoprotegerin expression and acceleration by high-dose insulin. Am J Physiol Heart Circ Physiol 2007;292:H1058-H1064.

32. Yuan LQ, Zhu JH, Wang HW, et al. RANKL is a downstream mediator for insulin-induced osteoblastic differentiation of vascular smooth muscle cells. PLoS One 2011;6:e29037.

33. Mandavia CH, Pulakat L, DeMarco V, Sowers JR. Over-nutrition and metabolic cardiomyopathy. Metabolism 2012;61:1205-1210.

34. Dhalla NS, Liu X, Panagia V, Takeda N. Subcellular remodeling and heart dysfunction in chronic diabetes. Cardiovasc Res 1998;40:239-247.

35. Liu J, Shen W, Zhao B, et al. Targeting mitochondrial bio- genesis for preventing and treating insulin resistance in diabetes and obesity: hope from natural mitochondrial nutrients. Adv Drug Deliv Rev 2009;61:1343-1352.

36. Falcao-Pires I, Leite-Moreira AF. Diabetic cardiomyopathy: understanding the molecular and cellular basis to progress in diagnosis and treatment. Heart Fail Rev 2012;17:325-344.

37. Adeghate E, Singh J. Structural changes in the myocardium during diabetes-induced cardiomyopathy. Heart Fail Rev 2014;19:15-23.

38. Schaffer SW. Cardiomyopathy associated with noninsulin-dependent diabetes. Mol Cell Biochem 1991;107:1-20.

39. Battiprolu PK, Lopez-Crisosto C, Wang ZV, Nemchenko A, Lavandero S, Hill JA. Diabetic cardiomyopathy and metabolic remodeling of the heart. Life Sci 2013;92:609-615.

40. Harmancey R, Lam TN, Lubrano GM, Guthrie PH, Vela $\mathrm{D}$, Taegtmeyer $\mathrm{H}$. Insulin resistance improves metabolic and contractile efficiency in stressed rat heart. FASEB J 2012;26:3118-3126.

41. McGavock JM, Lingvay I, Zib I, et al. Cardiac steatosis in diabetes mellitus: a $1 \mathrm{H}$-magnetic resonance spectroscopy study. Circulation 2007;116:1170-1175.

42. Rijzewijk LJ, van der Meer RW, Smit JW, et al. Myocardial steatosis is an independent predictor of diastolic dysfunction in type 2 diabetes mellitus. J Am Coll Cardiol 2008;52:1793-1799.

43. Rijzewijk LJ, van der Meer RW, Lamb HJ, et al. Altered myocardial substrate metabolism and decreased diastolic function in nonischemic human diabetic cardiomyopathy: studies with cardiac positron emission tomography and magnetic resonance imaging. J Am Coll Cardiol 2009;54:1524-1532.

44. van de Weijer T, Schrauwen-Hinderling VB, Schrauwen P. Lipotoxicity in type 2 diabetic cardiomyopathy. Cardiovasc Res 2011;92:10-18.

45. Balaban RS. Cardiac energy metabolism homeostasis: role of cytosolic calcium. J Mol Cell Cardiol 2002;34:12591271.

46. Iyngkaran P, Anavekar N, Majoni W, Thomas MC. The role and management of sympathetic overactivity in cardiovascular and renal complications of diabetes. Diabetes Metab 2013;39:290-298.

47. Olshansky B, Sabbah HN, Hauptman PJ, Colucci WS. Parasympathetic nervous system and heart failure: pathophysiology and potential implications for therapy. Circulation 2008;118:863-871. 
48. Pappachan JM, Sebastian J, Bino BC, et al. Cardiac autonomic neuropathy in diabetes mellitus: prevalence, risk factors and utility of corrected QT interval in the ECG for its diagnosis. Postgrad Med J 2008;84:205-210.

49. Di Carli MF, Bianco-Batlles D, Landa ME, et al. Effects of autonomic neuropathy on coronary blood flow in patients with diabetes mellitus. Circulation 1999;100:813819.

50. Taskiran M, Fritz-Hansen T, Rasmussen V, Larsson HB, Hilsted J. Decreased myocardial perfusion reserve in diabetic autonomic neuropathy. Diabetes 2002;51:3306-3310.

51. Erbas T, Erbas B, Kabakci G, Aksoyek S, Koray Z, Gedik O. Plasma big-endothelin levels, cardiac autonomic neuropathy, and cardiac functions in patients with insulin-dependent diabetes mellitus. Clin Cardiol 2000;23:259-263.

52. Kreiner G, Wolzt M, Fasching P, et al. Myocardial m-[123I] iodobenzylguanidine scintigraphy for the assessment of adrenergic cardiac innervation in patients with IDDM: comparison with cardiovascular reflex tests and relationship to left ventricular function. Diabetes 1995;44:543-549.

53. Kumar R, Yong QC, Thomas CM, Baker KM. Intracardiac intracellular angiotensin system in diabetes. Am J Physiol Regul Integr Comp Physiol 2012;302:R510-R517.

54. Kurdi M, Booz GW. New take on the role of angiotensin II in cardiac hypertrophy and fibrosis. Hypertension 2011;57:1034-1038.

55. Frustaci A, Kajstura J, Chimenti C, et al. Myocardial cell death in human diabetes. Circ Res 2000;87:1123-1132.

56. DeMarco VG, Aroor AR, Sowers JR. The pathophysiology of hypertension in patients with obesity. Nat Rev Endocrinol 2014;10:364-376.

57. McMaster WG, Kirabo A, Madhur MS, Harrison DG. Inflammation, immunity, and hypertensive end-organ damage. Circ Res 2015;116:1022-1033.

58. Hofmann U, Frantz S. Role of lymphocytes in myocardial injury, healing, and remodeling after myocardial infarction. Circ Res 2015;116:354-367.

59. Jia G, Habibi J, Bostick BP, et al. Uric acid promotes left ventricular diastolic dysfunction in mice fed a Western diet. Hypertension 2015;65:531-539.

6o. Mori J, Alrob OA, Wagg CS, Harris RA, Lopaschuk GD, Oudit GY. ANG II causes insulin resistance and induces cardiac metabolic switch and inefficiency: a critical role of PDK4. Am J Physiol Heart Circ Physiol 2013;304:H1103-H1113.

61. Asrih M, Mach F, Nencioni A, Dallegri F, Quercioli A,
Montecucco F. Role of mitogen-activated protein kinase pathways in multifactorial adverse cardiac remodeling associated with metabolic syndrome. Mediators Inflamm 2013;2013:367245.

62. Weirather J, Hofmann UD, Beyersdorf N, et al. Foxp3+ $\mathrm{CD}_{4}+\mathrm{T}$ cells improve healing after myocardial infarction by modulating monocyte/macrophage differentiation. Circ Res 2014;115:55-67.

63. Sell H, Habich C, Eckel J. Adaptive immunity in obesity and insulin resistance. Nat Rev Endocrinol 2012;8:709716.

64. Ait-Oufella H, Salomon BL, Potteaux S, et al. Natural regulatory $\mathrm{T}$ cells control the development of atherosclerosis in mice. Nat Med 2006;12:178-180.

65. Yu Q, Vazquez R, Zabadi S, Watson RR, Larson DF. T-lymphocytes mediate left ventricular fibrillar collagen cross-linking and diastolic dysfunction in mice. Matrix Biol 2010;29:511-518.

66. Cao Y, Xu W, Xiong S. Adoptive transfer of regulatory T cells protects against coxsackievirus B3-induced cardiac fibrosis. PLoS One 2013;8:e74955.

67. Voulgari C, Papadogiannis D, Tentolouris N. Diabetic cardiomyopathy: from the pathophysiology of the cardiac myocytes to current diagnosis and management strategies. Vasc Health Risk Manag 2010;6:883-903.

68. Nunes S, Soares E, Fernandes J, et al. Early cardiac changes in a rat model of prediabetes: brain natriuretic peptide overexpression seems to be the best marker. Cardiovasc Diabetol 2013;12:44.

69. Landsberg L, Molitch M. Diabetes and hypertension: pathogenesis, prevention and treatment. Clin Exp Hypertens 2004;26:621-628.

70. Mathis DR, Liu SS, Rodrigues BB, McNeill JH. Effect of hypertension on the development of diabetic cardiomyopathy. Can J Physiol Pharmacol 2000;78:791-798.

71. Aijaz B, Ammar KA, Lopez-Jimenez F, Redfield MM, Jacobsen SJ, Rodeheffer RJ. Abnormal cardiac structure and function in the metabolic syndrome: a population-based study. Mayo Clin Proc 2008;83:1350-1357.

72. Chavali V, Tyagi SC, Mishra PK. Predictors and prevention of diabetic cardiomyopathy. Diabetes Metab Syndr Obes 2013;6:151-160.

73. Ernande L, Derumeaux G. Diabetic cardiomyopathy: myth or reality? Arch Cardiovasc Dis 2012;105:218-225.

74. Battiprolu PK, Gillette TG, Wang ZV, Lavandero S, Hill JA. Diabetic cardiomyopathy: mechanisms and therapeutic 
targets. Drug Discov Today Dis Mech 2010;7:e135-e143.

75. Khouri SJ, Maly GT, Suh DD, Walsh TE. A practical approach to the echocardiographic evaluation of diastolic function. J Am Soc Echocardiogr 2004;17:290-297.

76. Yu CM, Sanderson JE, Marwick TH, Oh JK. Tissue Doppler imaging a new prognosticator for cardiovascular diseases. J Am Coll Cardiol 2007;49:1903-1914.

77. Miki T, Yuda S, Kouzu H, Miura T. Diabetic cardiomyopathy: pathophysiology and clinical features. Heart Fail Rev 2013;18:149-166.

78. Gottlieb I, Macedo R, Bluemke DA, Lima JA. Magnetic resonance imaging in the evaluation of non-ischemic cardiomyopathies: current applications and future perspectives. Heart Fail Rev 2006;11:313-323.

79. Paulus WJ, Tschope C, Sanderson JE, et al. How to diagnose diastolic heart failure: a consensus statement on the diagnosis of heart failure with normal left ventricular ejection fraction by the Heart Failure and Echocardiography Associations of the European Society of Cardiology. Eur Heart J 2007;28:2539-2550.

8o. Dinh W, Bansemir L, Futh R, et al. Increased levels of laminin and collagen type VI may reflect early remodelling in patients with acute myocardial infarction. Acta Cardiol 2009;64:329-334.

81. D'Souza A, Howarth FC, Yanni J, et al. Left ventricle structural remodelling in the prediabetic Goto-Kakizaki rat. Exp Physiol 2011;96:875-888.

82. Quilliot D, Alla F, Bohme P, et al. Myocardial collagen turnover in normotensive obese patients: relation to insulin resistance. Int J Obes (Lond) 2005;29:1321-1328.

83. Stolen TO, Hoydal MA, Kemi OJ, et al. Interval training normalizes cardiomyocyte function, diastolic $\mathrm{Ca}+$ control, and SR Ca2+ release synchronicity in a mouse model of diabetic cardiomyopathy. Circ Res 2009;105:527-536.

84. Epp RA, Susser SE, Morissette MP, Kehler DS, Jassal DS, Duhamel TA. Exercise training prevents the development of cardiac dysfunction in the low-dose streptozotocin diabetic rats fed a high-fat diet. Can J Physiol Pharmacol 2013;91:80-89.

85. Epshteyn V, Morrison K, Krishnaswamy P, et al. Utility of B-type natriuretic peptide (BNP) as a screen for left ventricular dysfunction in patients with diabetes. Diabetes Care 2003;26:2081-2087.

86. Russell NE, Higgins MF, Amaruso M, Foley M, McAuliffe FM. Troponin $\mathrm{T}$ and pro-B-type natriuretic peptide in fetuses of type 1 diabetic mothers. Diabetes Care
2009;32:2050-2055.

87. Feng B, Chen S, George B, Feng Q, Chakrabarti S. miR133a regulates cardiomyocyte hypertrophy in diabetes. Diabetes Metab Res Rev 2010;26:40-49.

88. Rijzewijk LJ, Jonker JT, van der Meer RW, et al. Effects of hepatic triglyceride content on myocardial metabolism in type 2 diabetes. J Am Coll Cardiol 2010;56:225-233.

89. Kodama S, Tanaka S, Heianza Y, et al. Association between physical activity and risk of all-cause mortality and cardiovascular disease in patients with diabetes: a meta-analysis. Diabetes Care 2013;36:471-479.

90. Sharma AK, Srinivasan BP. Triple verses glimepiride plus metformin therapy on cardiovascular risk biomarkers and diabetic cardiomyopathy in insulin resistance type 2 diabetes mellitus rats. Eur J Pharm Sci 2009;38:433-444.

91. Aboukhoudir F, Rekik S. Left ventricular systolic function deterioration during dobutamine stress echocardiography as an early manifestation of diabetic cardiomyopathy and reversal by optimized therapeutic approach. Int J Cardiovasc Imaging 2012;28:1329-1339.

92. Chung J, Abraszewski P, Yu X, et al. Paradoxical increase in ventricular torsion and systolic torsion rate in type I diabetic patients under tight glycemic control. J Am Coll Cardiol 2006;47:384-390.

93. Zib I, Jacob AN, Lingvay I, et al. Effect of pioglitazone therapy on myocardial and hepatic steatosis in insulin-treated patients with type 2 diabetes. J Investig Med 2007;55:230-236.

94. Xie Z, Lau K, Eby B, et al. Improvement of cardiac functions by chronic metformin treatment is associated with enhanced cardiac autophagy in diabetic OVE26 mice. Diabetes 2011;60:1770-1778.

95. von Bibra H, St John Sutton M. Impact of diabetes on postinfarction heart failure and left ventricular remodeling. Curr Heart Fail Rep 2011;8:242-251.

96. Wong AK, Symon R, AlZadjali MA, et al. The effect of metformin on insulin resistance and exercise parameters in patients with heart failure. Eur J Heart Fail 2012;14:13031310.

97. Mamas MA, Deaton C, Rutter MK, et al. Impaired glucose tolerance and insulin resistance in heart failure: underrecognized and undertreated? J Card Fail 2010;16:761-768.

98. Sacca L, Napoli R. Insulin resistance in chronic heart failure: a difficult bull to take by the horns. Nutr Metab Cardiovasc Dis 2009;19:303-305.

99. Caglayan E, Stauber B, Collins AR, et al. Differential roles 
of cardiomyocyte and macrophage peroxisome proliferator-activated receptor gamma in cardiac fibrosis. Diabetes 2008;57:2470-2479.

100. Younce CW, Burmeister MA, Ayala JE. Exendin-4 attenuates high glucose-induced cardiomyocyte apoptosis via inhibition of endoplasmic reticulum stress and activation of SERCA2a. Am J Physiol Cell Physiol 2013;304:C508-C518.

101. Doehner W, Frenneaux M, Anker SD. Metabolic impairment in heart failure: the myocardial and systemic perspective. J Am Coll Cardiol 2014;64:1388-1400.

102. Witteles RM, Keu KV, Quon A, Tavana H, Fowler MB. Dipeptidyl peptidase 4 inhibition increases myocardial glucose uptake in nonischemic cardiomyopathy. J Card Fail 2012;18:804-809.

103. Bostick B, Habibi J, Ma L, et al. Dipeptidyl peptidase inhibition prevents diastolic dysfunction and reduces myocardial fibrosis in a mouse model of Western diet induced obesity. Metabolism 2014;63:1000-1011.

104. Adeghate E, Kalasz H. Amylin analogues in the treatment of diabetes mellitus: medicinal chemistry and structural basis of its function. Open Med Chem J 2011;5:78-81.

105. Inzucchi SE, Zinman B, Wanner C, et al. SGLT-2 inhibitors and cardiovascular risk: proposed pathways and review of ongoing outcome trials. Diab Vasc Dis Res 2015;12:90-100.

106. Zinman B, Wanner C, Lachin JM, et al. Empagliflozin, cardiovascular outcomes, and mortality in type 2 diabetes. N Engl J Med 2015;373:2117-2128.

107. Thomas CM, Yong QC, Seqqat R, et al. Direct renin inhibition prevents cardiac dysfunction in a diabetic mouse model: comparison with an angiotensin receptor antagonist and angiotensin-converting enzyme inhibitor. Clin Sci (Lond) 2013;124:529-541.

108. Machackova J, Liu X, Lukas A, Dhalla NS. Renin-angiotensin blockade attenuates cardiac myofibrillar remodelling in chronic diabetes. Mol Cell Biochem 2004;261:271-278.

109. Symeonides P, Koulouris S, Vratsista E, et al. Both ramipril and telmisartan reverse indices of early diabetic cardiomyopathy: a comparative study. Eur J Echocardiogr 2007;8:480-486.

110. Sharma V, McNeill JH. Parallel effects of $\beta$-adrenoceptor blockade on cardiac function and fatty acid oxidation in the diabetic heart: confronting the maze. World J Cardiol 2011;3:281-302.

111. Mohamad HE, Askar ME, Hafez MM. Management of cardiac fibrosis in diabetic rats: the role of peroxisome proliferator activated receptor gamma (PPAR-gamma) and calcium channel blockers (CCBs). Diabetol Metab Syndr 2011;3:4.

112. Giannetta E, Isidori AM, Galea N, et al. Chronic inhibition of cGMP phosphodiesterase $5 \mathrm{~A}$ improves diabetic cardiomyopathy: a randomized, controlled clinical trial using magnetic resonance imaging with myocardial tagging. Circulation 2012;125:2323-2333.

113. Chen YH, Feng B, Chen ZW. Statins for primary prevention of cardiovascular and cerebrovascular events in diabetic patients without established cardiovascular diseases: a meta-analysis. Exp Clin Endocrinol Diabetes 2012;120:116-120.

114. Cholesterol Treatment Trialists' (CTT) Collaborators, Kearney PM, Blackwell L, et al. Efficacy of cholesterol-lowering therapy in 18,686 people with diabetes in 14 randomised trials of statins: a meta-analysis. Lancet 2008;371:117-125.

115. Van Linthout S, Riad A, Dhayat N, et al. Anti-inflammatory effects of atorvastatin improve left ventricular function in experimental diabetic cardiomyopathy. Diabetologia 2007;50:1977-1986.

116. Dai QM, Lu J, Liu NF. Fluvastatin attenuates myocardial interstitial fibrosis and cardiac dysfunction in diabetic rats by inhibiting over-expression of connective tissue growth factor. Chin Med J (Engl) 2011;124:89-94.

117. Nickel A, Loffler J, Maack C. Myocardial energetics in heart failure. Basic Res Cardiol 2013;108:358.

118. Gao D, Ning N, Niu X, Hao G, Meng Z. Trimetazidine: a meta-analysis of randomised controlled trials in heart failure. Heart 2011;97:278-286.

119. Zhao P, Zhang J, Yin XG, et al. The effect of trimetazidine on cardiac function in diabetic patients with idiopathic dilated cardiomyopathy. Life Sci 2013;92:633-638.

120. Li YJ, Wang PH, Chen C, Zou MH, Wang DW. Improvement of mechanical heart function by trimetazidine in $\mathrm{db} / \mathrm{db}$ mice. Acta Pharmacol Sin 2010;31:560-569.

121. Maier LS, Layug B, Karwatowska-Prokopczuk E, et al. RAnoLazIne for the treatment of diastolic heart failure in patients with preserved ejection fraction: the RALI-DHF proof-of-concept study. JACC Heart Fail 2013;1:115-122.

122. Senanayake EL, Howell NJ, Ranasinghe AM, et al. Multicentre double-blind randomized controlled trial of perhexiline as a metabolic modulator to augment myocardial protection in patients with left ventricular hypertrophy 
undergoing cardiac surgery. Eur J Cardiothorac Surg 2015;48:354-362.

123. Li CJ, Lv L, Li H, Yu DM. Cardiac fibrosis and dysfunction in experimental diabetic cardiomyopathy are ameliorated by alpha-lipoic acid. Cardiovasc Diabetol 2012;11:73.

124. Delucchi F, Berni R, Frati C, et al. Resveratrol treatment reduces cardiac progenitor cell dysfunction and prevents morpho-functional ventricular remodeling in type-1 diabetic rats. PLoS One 2012;7:e39836.

125. Wang G, Li W, Lu X, Bao P, Zhao X. Luteolin ameliorates cardiac failure in type I diabetic cardiomyopathy. J Diabetes Complications 2012;26:259-265.

126. Wang G, Li W, Lu X, Zhao X. Riboflavin alleviates cardiac failure in type I diabetic cardiomyopathy. Heart Int 2011;6:e21.

127. Xu X, Xiao H, Zhao J, Zhao T. Cardioprotective effect of sodium ferulate in diabetic rats. Int J Med Sci 2012;9:291300.

128. Sulaiman M, Matta MJ, Sunderesan NR, Gupta MP, Periasamy M, Gupta M. Resveratrol, an activator of SIRT1, upregulates sarcoplasmic calcium ATPase and improves cardiac function in diabetic cardiomyopathy. Am J Physiol Heart Circ Physiol 2010;298:H833-H843.

129. Rabassa M, Zamora-Ros R, Urpi-Sarda M, Andres-Lacueva C. Resveratrol metabolite profiling in clinical nutrition research: from diet to uncovering disease risk biomarkers: epidemiological evidence. Ann N Y Acad Sci 2015;1348:107-115.

130. Xu YJ, Tappia PS, Neki NS, Dhalla NS. Prevention of diabetes-induced cardiovascular complications upon treatment with antioxidants. Heart Fail Rev 2014;19:113-121.

131. Szeto HH. First-in-class cardiolipin-protective compound as a therapeutic agent to restore mitochondrial bioenergetics. Br J Pharmacol 2014;171:2029-2050.

132. Huynh K, Kiriazis H, Du XJ, et al. Coenzyme Q10 attenuates diastolic dysfunction, cardiomyocyte hypertrophy and cardiac fibrosis in the $\mathrm{db} / \mathrm{db}$ mouse model of type 2 diabetes. Diabetologia 2012;55:1544-1553.

133. Mortensen SA, Rosenfeldt F, Kumar A, et al. The effect of coenzyme Q10 on morbidity and mortality in chronic heart failure: results from Q-SYMBIO: a randomized double-blind trial. JACC Heart Fail 2014;2:641-649.

134. Huynh K, Bernardo BC, McMullen JR, Ritchie RH. Diabetic cardiomyopathy: mechanisms and new treatment strategies targeting antioxidant signaling pathways. Pharmacol Ther 2014;142:375-415.

135. Meloni M, Descamps B, Caporali A, et al. Nerve growth factor gene therapy using adeno-associated viral vectors prevents cardiomyopathy in type 1 diabetic mice. Diabetes 2012;61:229-240.

136. Katare R, Caporali A, Zentilin L, et al. Intravenous gene therapy with PIM-1 via a cardiotropic viral vector halts the progression of diabetic cardiomyopathy through promotion of prosurvival signaling. Circ Res 2011;108:12381251.

137. Greco S, Fasanaro P, Castelvecchio S, et al. MicroRNA dysregulation in diabetic ischemic heart failure patients. Diabetes 2012;61:1633-1641.

138. Chen H, Untiveros GM, McKee LA, et al. Micro-RNA-195 and -451 regulate the $\mathrm{LKB} 1 / \mathrm{AMPK}$ signaling axis by targeting MO25. PLoS One 2012;7:e41574.

139. Cheng Y, Guo S, Liu G, et al. Transplantation of bone marrow-derived endothelial progenitor cells attenuates myocardial interstitial fibrosis and cardiac dysfunction in streptozotocin-induced diabetic rats. Int J Mol Med 2012;30:870-876. 